\title{
Effects of Food Consistencies and Mastication on Bolus Transport and Swallow Initiation in Individuals with Hemispheric Stroke
}

Koichiro Matsuo ${ }^{1,2^{\star}}$, Michio Yokoyama ${ }^{1}$, Marlis Gonzalez-Fernandez ${ }^{3}$, Eiichi Saitoh ${ }^{1}$, Hitoshi Kagaya ${ }^{1}$, Mikoto Baba ${ }^{1}$, Wataru Fujii ${ }^{1,4}$ and Jeffrey B. Palmer $^{3,5}$

${ }^{1}$ Department of Rehabilitation Medicine I, School of Medicine, Fujita Health University, Aichi, Japan

${ }^{2}$ Department of Dentistry, School of Medicine, Fujita Health University, Aichi, Japan

${ }^{3}$ Department of Physical Medicine and Rehabilitation, Johns Hopkins University, Baltimore, MD, USA

${ }^{4}$ Department of Dental Surgery, Fujita Health University Nanakuri Sanatorium, Mie, Japan

${ }^{5}$ Department of Otolaryngology-Head and Neck Surgery, and Center for Functional Anatomy and Evolution, Johns Hopkins University, Baltimore, MD, USA

*Correspondence author: Koichiro Matsuo, Professor and Chair, Department of Dentistry, School of Medicine, Fujita Health University, 1-98 Dengakugakubo, Kutsukake Toyoake, Aichi 470-1192, Japan; Tel: +81-562-93-9097; Fax: +81-562-93-2209, E-mail: kmatsuo@fujita-hu.ac.jp

Received date: Nov 26, 2014, Accepted date: Feb 16, 2015, Published date: Feb 22, 2015

Copyright: $\odot 2015$ Matsuo K, et al. This is an open-access article distributed under the terms of the Creative Commons Attribution License, which permits unrestricted use, distribution, and reproduction in any medium, provided the original author and source are credited.

Abstract
Mastication and food consistency each affect the passage of food through the oral cavity and pharynx and its
coordination with swallow initiation. Since hemispheric stroke may damage cortico-medullary neural pathways, we
hypothesized that it could also alter the coordination of eating and swallowing. Using videofluorography, we studied
30 individuals with hemispheric stroke and 27 age-matched controls as they consumed liquid barium, corned beef
hash, or a combination of liquid and solid (two-phase) food. We tracked the leading edge of the barium during
feeding and at swallow onset in relation to occurrences of laryngeal penetration and aspiration. Compared with
asymptomatic controls, the leading edge remained in the hypopharynx before swallowing longer in stroke subjects
(P $=0.016)$ and was lower in the foodway at swallow onset with two-phase food ( $P=0.03)$ and liquid ( $P=0.06)$.
Penetration-aspiration scores were significantly higher for liquid and two-phase ingesta when the leading edge was
deeper in the pharynx at swallow onset $(P=0.03)$. These findings suggest that stroke alters the coordination of
bolus transport and swallowing, particularly with foods containing a liquid phase. With such an impairment, a bolus
may enter the hypopharynx prematurely, thus increasing the risk of aspiration.

Keywords: Deglutition; Deglutition Disorders; Cerebral Stroke; Mastication; Rheology; Fluoroscopy

\begin{abstract}
Abbreviations
VFG, Videofluorography; OC, oral cavity; UOP, upper oropharynx; VAL, valleculae; HYP, hypopharynx; FPS, floor of piriform sinus; OCT, oral cavity time; PFAT, post-faucial aggregation time; VAT, vallecular aggregation time; HTT, hypopharyngeal transit time
\end{abstract}

\section{Introduction}

Dysphagia is common following hemispheric stroke [1]. The incidence of dysphagia in stroke survivors ranges from approximately $30 \%$ when evaluated by screening to up to $70 \%$ using instrumental assessments [2-5]. The specific mechanism of swallowing dysfunction after unilateral hemispheric stroke remains unclear, although studies suggest that hemispheric stroke likely damages the neural pathways from the cerebral cortex to the medullary swallowing centers [6,7]. Damage to neural pathways may hamper motor control of oral and pharyngeal structures or alter their afferent inputs. Swallowing impairments due to stroke include reduced lingual control, delayed swallowing reflex, reduced pharyngeal peristalsis, and oropharyngeal sensory deficits [8-12]. These dysfunctions are suspected contributors to the occurrence of aspiration.

Stage transition duration, defined as the duration from the moment at which the bolus passes the ramus of the mandible until maximal excursion of the hyoid is initiated, is longer in dysphagic stroke survivors than in normal subjects [8]. However, several studies have shown that barium samples entering the pharynx before swallow onset is not always abnormal; pre-swallow bolus entry into the pharynx can also occur in healthy individuals while drinking liquid [13-15]. Other reports have described that food can enter the pharynx up to $10 \mathrm{~s}$ before swallowing when healthy adults eat solid food [16-18]. During eating, the chewed bolus is transported to the oropharynx and valleculae well before swallow initiation $[16,19]$. When healthy normal adults eat food having two phases (i.e., a liquid and solid), a portion of the food can reach the hypopharynx before swallow initiation in $60 \%$ of cases [17].

While the presence of the food in the valleculae or hypopharynx before swallowing is not necessarily abnormal, it increases the risk of aspiration in individuals with dysphagia and impaired airway protection. The swallowing of liquid and semi-solid food has been studied in individuals with dysphagia due to stroke $[8,11,20,21]$ but little is known about how stroke affects the eating and swallowing of two-phase food. As such, the aim of the present study was to examine the impact of stroke on oral and pharyngeal food transport and swallow initiation while stroke survivors ate of two-phase food. Our primary hypothesis was that the leading edge of the food entered the pharynx earlier, and was deeper in the pharynx at swallow onset in stroke survivors with dysphagia than in healthy age-matched controls. Our secondary hypothesis was that deeper location of the leading edge of the food at swallow initiation was associated with an increased risk of aspiration through the vocal folds. 
Citation: Matsuo K, Yokoyama M, Gonzalez-Fernandez M, Saitoh E, Kagaya H, et al. (2015) Effects of Food Consistencies and Mastication on Bolus Transport and Swallow Initiation in Individuals with Hemispheric Stroke. J Neurol Neurophysiol 6: 269. doi: $10.4172 / 2155-9562.1000269$

Page 2 of 9

\section{Materials and Methods}

This study's protocol was approved by the Institutional Review Board. Prior to enrolment, the subjects received detailed information on the study and provided oral and written informed consent.

\section{Selection of stroke subjects}

Study participants were selected from a tertiary-care academic medical center in Japan. Subjects with dysphagia due to first unilateral ischemic or hemorrhagic hemispheric stroke with no brainstem lesions in computed tomography and/or magnetic resonance imaging were included. Individuals who had experienced tracheostomy, recurrent stroke, brainstem or bilateral hemispheric stroke, or cognitive deficits (unable to follow instructions) were excluded. As a result, thirty individuals with first hemispheric stroke (21 males and 9 females) whose median age was 69.5 years old (interquartile range: 63 - 80 years) participated in this study after neurological evaluation by physiatrists. Videofluorographic (VFG) examination of swallowing was performed a median 35 days (interquartile range: 21 - 60 days) after the onset of hemispheric stroke.

\section{Selection of control subjects}

Twenty-seven age-matched healthy asymptomatic elderly individuals (19 males and 8 females) were recruited as controls (median age: 68.0 years; interquartile range: 61 - 73 years). Participants in the control group had no history of neurological or laryngopharyngeal disorders that may have caused dysphagia and no complaints of feeding or swallowing problems. All subjects ingested their regular meals normally and were independent in performing activities of daily living.

\section{Procedure}

The VFG procedure has been reported previously [17]. Each subject was seated comfortably in an upright examination chair. VFG was recorded in the lateral projection with a digital videotape recorder at 30 frames/s from ingestion until terminal swallow. Each subject consumed three test foods: 1) $10 \mathrm{ml}$ of $50 \% \mathrm{wt} / \mathrm{vol}$ liquid barium; 2) 8 $\mathrm{g}$ of corned beef hash mixed with barium powder (a semi-solid food); and 3) a combination of $4 \mathrm{~g}$ of corned beef hash with $5 \mathrm{ml}$ of liquid barium (a two-phase food - having liquid and semisolid components). The foods were placed in the mouth by a physiatrist. A water syringe was used to place the liquid barium into the mouth. Corned beef hash was placed into the mouth with a spoon. For two-phase food, the corned beef hash was placed into the mouth first and immediately followed by injection of the liquid barium. The subjects were instructed to swallow in their usual manner for each food consistency.

\section{Data reduction}

VFG recordings were reviewed both in slow motion and frame-byframe. We analyzed only the first sequence in each recording (from the time the food entered the mouth until the completion of the first swallow). From an initial 171 recordings, we excluded 24 from the analysis: six recordings of two-phase food ingestion in the stroke group because the liquid barium was swallowed before the start of mastication; 18 recordings because of limited image quality (seven in the control group and eleven in the stroke group). One subject in the stroke group, who aspirated corned beef hash, was excluded because of phenomenology very different from the others. This subject retained corned beef hash in the valleculae for 65 seconds while mastication continued. Saliva mixed with the barium and entered the hypopharynxwhile most of the bolus remained in the valleculae. The mixture in the hypopharynx was subsequently aspirated through the vocal folds. After these exclusions, 147 recordings (74 recordings of stroke subjects and 73 of controls) were included in the analysis.

To measure the movement of the leading edge of the food, ingestion was divided into four stages as described previously $[17,19]$ :

(1) Oral Cavity Time (OCT): the time from when food was placed into the mouth until the leading edge of the barium reached the posterior nasal spine;

(2) Post-Faucial Aggregation Time (PFAT): the time from the end of OCT until the leading edge of the barium reached the lower border of the mandible;

(3) Vallecular Aggregation Time (VAT): the time from the end of PFAT until the leading edge of the barium passed the edge of the epiglottis; and

(4) Hypopharyngeal Transit Time (HTT): the time from the end of VAT until the trailing edge of the barium passed the upper esophageal sphincter.

We also noted the location of the leading edge of the barium at the moment of swallow initiation. Swallow initiation was defined as the moment when the hyoid bone started its rapid upward and forward elevation. Leading edge location was classified into one of the following five areas (Figure 1): (1) the oral cavity (from the lips to the posterior nasal spine); (2) the upper oropharynx (behind the posterior nasal spine but above the level of the lower border of the mandible); (3) the valleculae (below the lower border of the mandible but above the edge of the epiglottis); (4) the hypopharynx (below the epiglottis but above the floor of the piriform sinus); and (5) the floor of the piriform sinus.

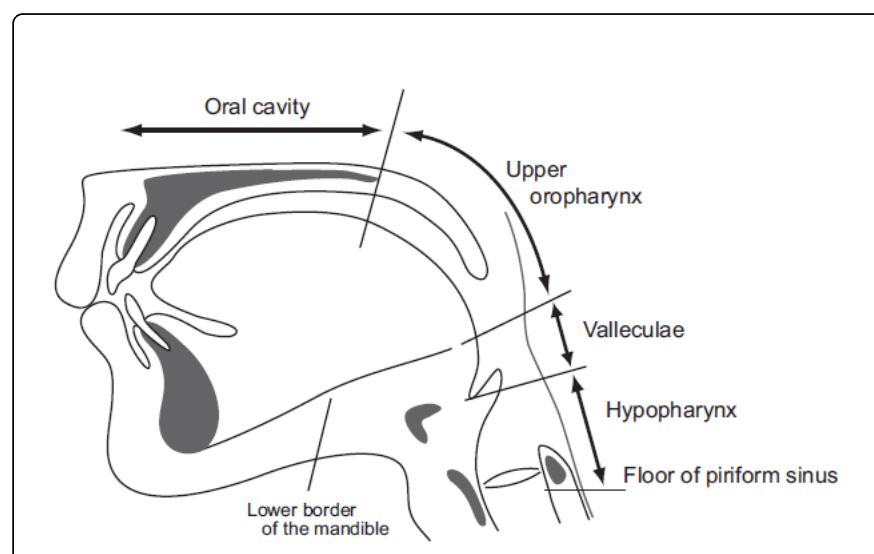

Figure 1: Location of the leading edge of the barium was defined by five anatomical regions: (1) the oral cavity; (2) the upper oropharynx behind the fauces but above the inferior border of the mandible; (3) the valleculae below the inferior border of the mandible but above the hypopharynx; (4) the hypopharynx below the epiglottis but not reaching the floor of the piriform sinus; and (5) the floor of the piriform sinus. 
Citation: Matsuo K, Yokoyama M, Gonzalez-Fernandez M, Saitoh E, Kagaya H, et al. (2015) Effects of Food Consistencies and Mastication on Bolus Transport and Swallow Initiation in Individuals with Hemispheric Stroke. J Neurol Neurophysiol 6: 269. doi: $10.4172 / 2155-9562.1000269$

Page 3 of 9

The presence of laryngeal penetration and/or aspiration through the vocal folds was scored for each swallow using the Rosenbek Penetration-Aspiration (PA) scale (Table 2) [22]. The PA scale is an 8- point ordinal scale based on the depth of material entry into the airway and the response elicited, where 1 indicates no airway invasion, 2-5 signifies laryngeal penetration, and 6-8 indicates aspiration.

\begin{tabular}{|c|c|c|c|c|c|c|c|c|c|c|c|}
\hline \multicolumn{12}{|l|}{ Stroke group } \\
\hline & \multirow{2}{*}{$\begin{array}{l}\text { PA } \\
\text { scale }\end{array}$} & \multicolumn{2}{|c|}{ OC } & \multicolumn{2}{|c|}{ UOP } & \multicolumn{2}{|c|}{ VAL } & \multicolumn{2}{|c|}{ HYP } & \multicolumn{2}{|c|}{ FPS } \\
\hline & & $\mathrm{n}$ & $(\%)$ & $\mathrm{n}$ & $(\%)$ & $n$ & $(\%)$ & $\mathrm{n}$ & $(\%)$ & $\mathrm{N}$ & $(\%)$ \\
\hline Liquid & 1 & & & 2 & -8.3 & 3 & -12.5 & 1 & -4.2 & 3 & -12.5 \\
\hline \multirow[t]{6}{*}{$(n=24)$} & 2 & & & 1 & -4.2 & 1 & -4.2 & 2 & -8.3 & 1 & -4.2 \\
\hline & 3 & & & & & & & 1 & -4.2 & 2 & -8.3 \\
\hline & 4 & & & & & & & & & 1 & -4.2 \\
\hline & 5 & & & 1 & -4.2 & & & 1 & -4.2 & 2 & -8.3 \\
\hline & 6 & & & & & & & & & 1 & -4.2 \\
\hline & 8 & & & & & & & & & 1 & -4.2 \\
\hline Corned beef hash & 1 & & & 7 & -26.9 & 11 & -42.3 & 2 & -7.7 & & \\
\hline \multirow[t]{2}{*}{$(n=26)$} & 2 & & & & & 1 & -3.8 & & & & \\
\hline & 3 & & & & & 3 & -11.5 & 2 & -7.7 & & \\
\hline Two-phase & 1 & & & 1 & -4.8 & & & 1 & -4.8 & 4 & -19 \\
\hline \multirow[t]{4}{*}{$(n=21)$} & 2 & & & & & & & 2 & -9.5 & 3 & -14.3 \\
\hline & 3 & & & & & 1 & -4.8 & & & 5 & -23.8 \\
\hline & 5 & & & & & & & & & 1 & -4.8 \\
\hline & 8 & & & & & & & & & 3 & -14.3 \\
\hline \multicolumn{12}{|l|}{ Control group } \\
\hline \multirow[t]{3}{*}{ Liquid ( $n=24)$} & 1 & 1 & -4.2 & 3 & -12.5 & 3 & -12.5 & 4 & -16.7 & 4 & -16.7 \\
\hline & 2 & & & 2 & -8.3 & 3 & -12.5 & 1 & -4.2 & 1 & -4.2 \\
\hline & 5 & & & 1 & -4.2 & 1 & -4.2 & & & & \\
\hline Corned beef hash & 1 & 1 & -4 & 4 & -16 & 19 & -76 & 1 & -4 & & \\
\hline \multicolumn{12}{|l|}{$(n=25)$} \\
\hline \multirow[t]{4}{*}{ Two-phase $(n=23)$} & 1 & 1 & -4.3 & 1 & -4.3 & 3 & -13 & 7 & -30.4 & 3 & -13 \\
\hline & 2 & & & & & 1 & -4.3 & & & 5 & -21.7 \\
\hline & 3 & & & & & & & & & 1 & -4.3 \\
\hline & 5 & & & & & & & & & 1 & -4.3 \\
\hline
\end{tabular}

Table 2: Relationship between location of leading edge and Penetration-Aspiration scalescore

PA scale, Penetration-Aspiration scale; OC, oral cavity; UOP, upper oropharynx; VAL, valleculae; HYP, hypopharynx; FPS, floor of piriform sinus

\section{Data analysis}

The effects of stroke and food type on duration of eating stage (OCT to HTT) and on the interval from the start and end of HTT to swallow onset were tested. Since the variances of the duration measures were not homogenous and increased with higher mean values, the data were first logarithmically transformed for statistical analysis. To test the effect of group (stroke vs. control) and food type (liquid, corned beef hash, or two-phase) on the log-transformed duration measures, we employed two-way ANOVA by food type (intra-subject effects) and group (inter-subject effects). Bonferroni correction was used for multiple comparisons. 
Citation: Matsuo K, Yokoyama M, Gonzalez-Fernandez M, Saitoh E, Kagaya H, et al. (2015) Effects of Food Consistencies and Mastication on Bolus Transport and Swallow Initiation in Individuals with Hemispheric Stroke. J Neurol Neurophysiol 6: 269. doi: $10.4172 / 2155-9562.1000269$

Page 4 of 9

The Mann-Whitney U test was used to compare the location of the leading edge of the barium at swallow onset between stroke and control groups for each food sample. PA scale scores for each swallow were tested using ANOVA by food type, subject group, and location of the leading edge at swallow onset.

The critical value for rejecting the null hypothesis was $\mathrm{P}<0.05$. Statistical procedures were performed with SPSS 12.0 software (SPSS Inc., Chicago, IL).

\section{Results}

\section{Characteristics of stroke subjects}

The demographic characteristics of the stroke subjects are summarized in Table 1. Most had experienced ischemic stroke $(73.3 \%)$. More than $50 \%$ of lesions were in the basal ganglia or internal capsule. There were similar numbers of right and left hemisphere strokes. Most subjects used modifications of feeding because of their dysphagia: $20 \%$ received tube feeding only, $10 \%$ received combined tube and oral feedings, and $56.7 \%$ were on a modified diet.

\begin{tabular}{|c|c|c|}
\hline & $\mathrm{n}$ & (\%) \\
\hline \multicolumn{3}{|l|}{ Type of stroke } \\
\hline Infarction & 22 & -73.3 \\
\hline Hemorrhage & 8 & -26.7 \\
\hline \multicolumn{3}{|l|}{ Location of lesion } \\
\hline Basal ganglia and/or internal capsule & 16 & -53.3 \\
\hline Thalami & 1 & -3.3 \\
\hline Corona radiata & 3 & -10 \\
\hline
\end{tabular}

\begin{tabular}{|l|l|l|}
\hline Insula & 3 & -10 \\
\hline Extensive lesions & 2 & -6.7 \\
\hline Others & 5 & -16.7 \\
\hline Side of lesion & \multicolumn{2}{|l|}{} \\
\hline Right hemisphere & 14 & -46.7 \\
\hline Left hemisphere & 16 & -53.3 \\
\hline Status of ingestion & \multicolumn{2}{|l|}{} \\
\hline Tube feeding only & 6 & -20 \\
\hline Tube > oral feeding & 2 & -6.7 \\
\hline Tube < oral feeding & 2 & -3.3 \\
\hline Modified food & 17 & -56.7 \\
\hline General diet & 4 & -13.3 \\
\hline
\end{tabular}

Table 1: Characteristics of stroke subjects

\section{Location of the leading edge of the barium at swallow onset (Figure 2)}

For liquid swallows, the leading edge of the barium was deeper in the pharynx at swallow onset in the stroke group than in controls; this difference approached statistical significance $(P=0.057)$. The leading edge was in the hypopharyngeal areas at swallow onset in $68 \%$ of stroke subjects (20\% in the hypopharynx and $48 \%$ at the floor of the piriform sinus) but only in $42 \%$ of controls ( $21 \%$ in the hypopharynx and $21 \%$ at the floor of the piriform sinus). 
Citation: Matsuo K, Yokoyama M, Gonzalez-Fernandez M, Saitoh E, Kagaya H, et al. (2015) Effects of Food Consistencies and Mastication on Bolus Transport and Swallow Initiation in Individuals with Hemispheric Stroke. J Neurol Neurophysiol 6: 269. doi: $10.4172 / 2155-9562.1000269$

Page 5 of 9

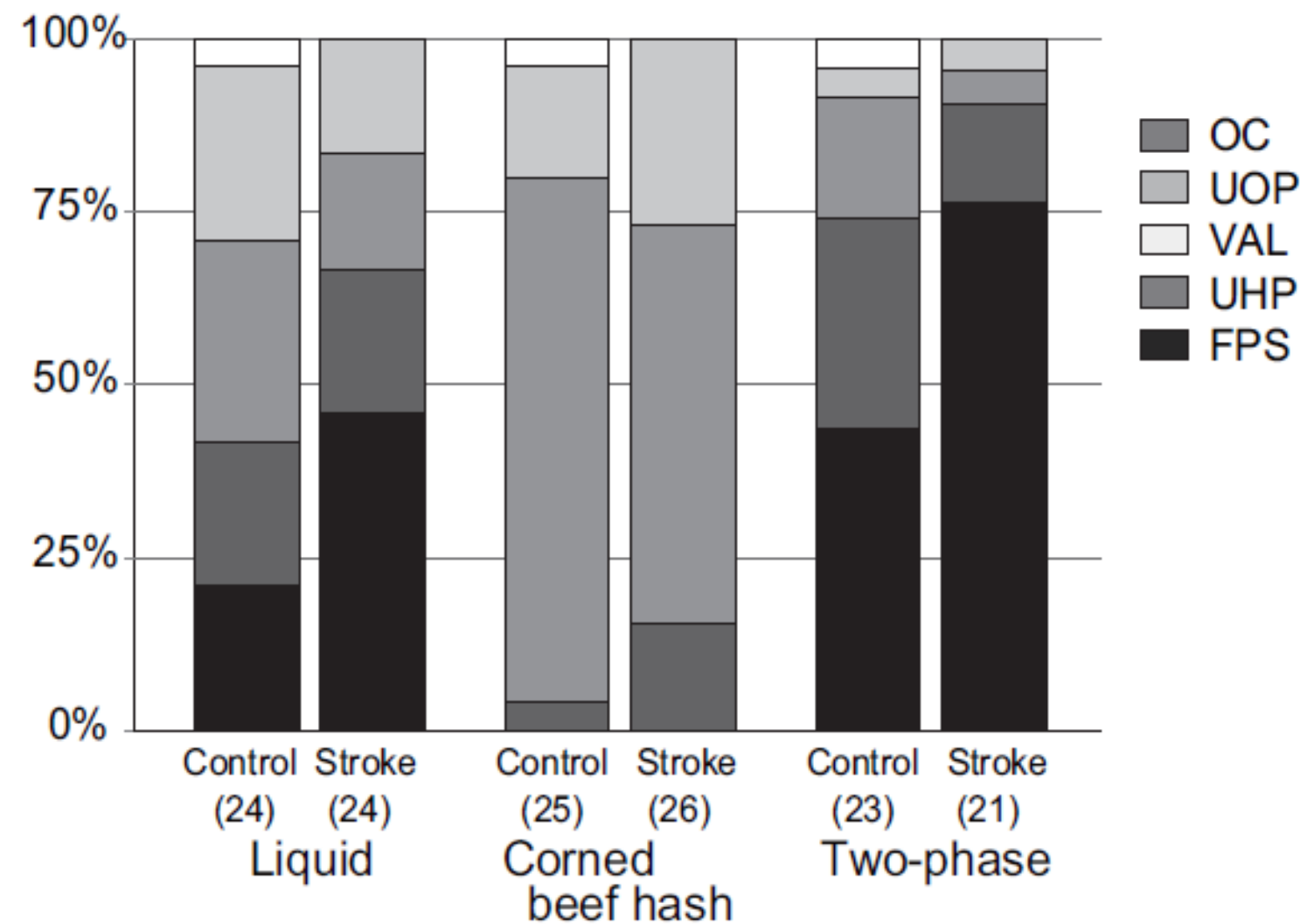

Figure 2: Percentage bar graph of the location of the leading edge of the barium at swallow onset for each food type in stroke and control subjects. For liquid and two-phase food, the leading edge was lower in the foodway at swallow onset in the stroke group than with in controls $(\mathrm{P}=0.057$ for liquid and $\mathrm{P}=0.03$ for two-phase). For corned beef hash, the leading edge was usually in the oropharynx in both groups $(\mathrm{P}=$ 0.80). The number of recordings is shown in parentheses for each food type. OC, oral cavity; UOP, upper oropharynx; VAL, valleculae; HYP, hypopharynx; FPS, floor of piriform sinus

With two-phase food, the leading edge of the barium was significantly deeper in the pharynx at swallow onset in stroke subjects than in controls $(\mathrm{P}=0.03)$. The leading edge was usually below the epiglottis at swallow onset in both stroke and control groups $(91 \%$ and $74 \%$, respectively). However, of these, the leading edge was at the floor of the piriform sinus at swallow onset in $76 \%$ of stroke group individuals as compared with $44 \%$ of controls.

With corned beef hash, the leading edge of the barium was in the oropharynx, (including the upper oropharynx and valleculae) in $92 \%$ of controls and $85 \%$ of stroke subjects at swallow onset $(P=0.80)$. The leading edge infrequently entered the hypopharynx and in no case reached the floor of the piriform sinus before swallowing.

\section{Laryngeal penetration and aspiration}

In the control group, laryngeal penetration was observed in $38 \%$ of the recordings with liquid and $35 \%$ with two-phase food, though the location of the leading edge of the barium at swallow onset differed significantly. With liquid, laryngeal penetration occurred when the leading edge was in the oropharynx at swallow onset (three cases in the upper oropharynx and four cases in the valleculae). On the other hand, with two-phase food, penetration occurred when the leading edge reached the floor of the piriform sinus before swallow onset. Only one control subject displayed penetration when the leading edge reached the valleculae. Aspiration was not observed in the control group.

In the stroke group, laryngeal penetration was observed in $54 \%$ of the recordings with liquid, 23\% with corned beef hash, and 57\% with two-phase samples. With liquid and two-phase samples, the incidence of penetration was higher when the leading edge of the barium was at a lower position at swallow onset. When laryngeal penetration occurred with corned beef hash, the leading edge was in the valleculae (four cases) or hypopharynx (two cases). Five instances of aspiration were observed in four stroke subjects: two with liquid and three with twophase food. The leading edge was at the floor of the piriform sinus at swallow onset in all cases of aspiration.

The location of the leading edge of the barium at swallow onset had a significant effect on PA scale scores $(\mathrm{P}=0.027)$. PA scale scores also differed significantly between stroke subjects and controls $(\mathrm{P}=0.002)$ (Table 2). PA scale score was significantly higher when the leading edge was at the floor of the piriform sinus at swallow onset $(\mathrm{P}<0.003)$. Food type did not significantly affect PA scale score $(\mathrm{P}=0.21)$. There were no significant interactions between leading edge position and food type $(\mathrm{P}=0.80)$ or subject group $(\mathrm{P}=0.072)$. 
Citation: Matsuo K, Yokoyama M, Gonzalez-Fernandez M, Saitoh E, Kagaya H, et al. (2015) Effects of Food Consistencies and Mastication on Bolus Transport and Swallow Initiation in Individuals with Hemispheric Stroke. J Neurol Neurophysiol 6: 269. doi: $10.4172 / 2155-9562.1000269$

Page 6 of 9

Movement of the leading edge of the barium during feeding and swallowing (Figure 3, 4)

The durations of OCT, PFAT, and VAT did not differ remarkably between stroke subjects and controls but were significantly different among food types $(\mathrm{P}<0.001$ for each stage) (Figure 3$)$. Each of the OCT, PFAT, and VAT durations were significantly longer with corned beef hash than with the other foods $(\mathrm{P}<0.02)$. OCT and PFAT did not differ significantly between two-phase and liquid samples.

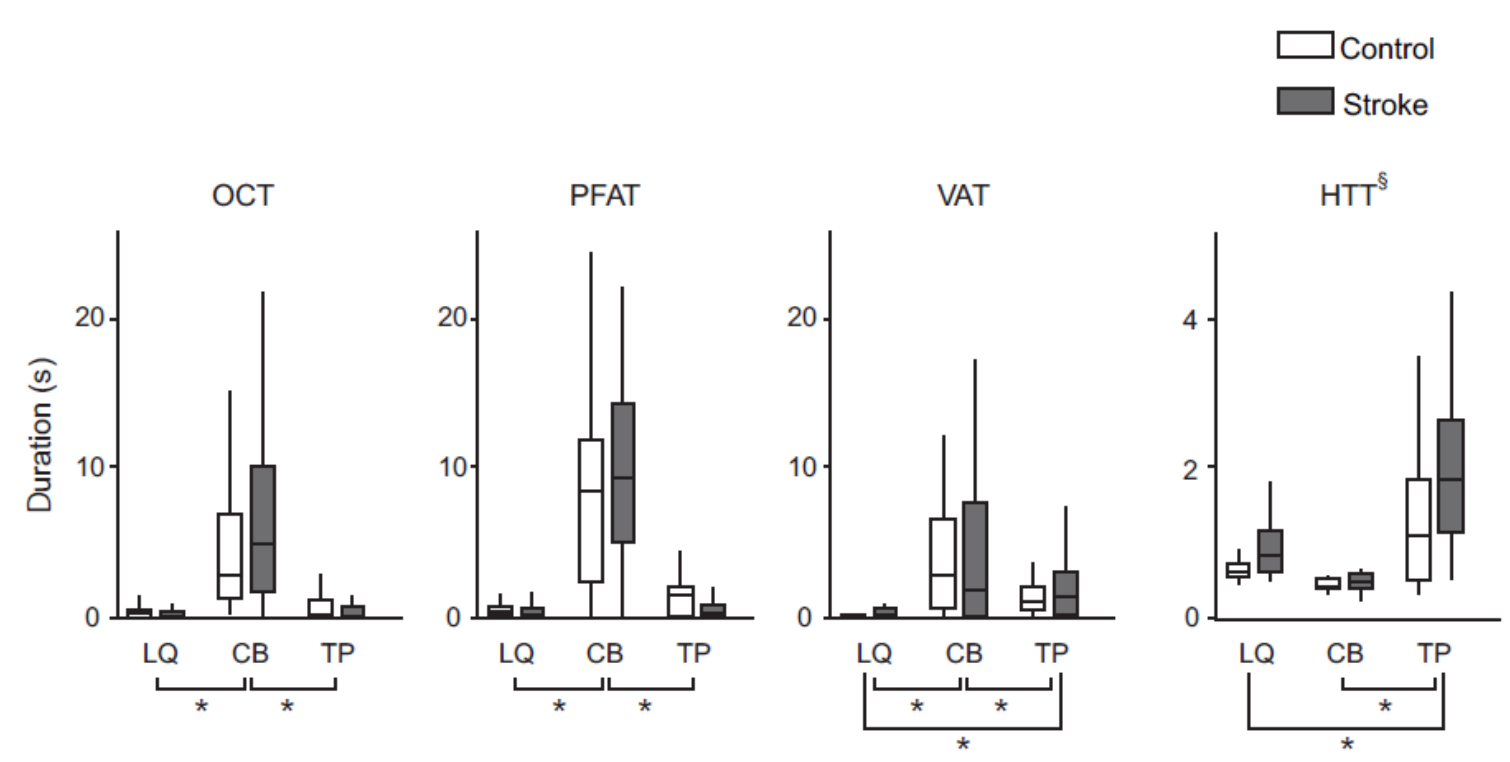

Figure 3: Stage duration by food and subject group. The durations of OCT, PFAT, and VAT were significantly longer with the semi-solid food than with the other foods. HTT duration was significantly longer with the two-phase food than with the other foods and was significantly longer in the stroke group than in controls. OCT, oral cavity time; PFAT, post-faucial aggregation time; VAT, vallecular aggregation time; HTT, hypopharyngeal transit time; LQ, liquid; $\mathrm{CB}$, corned beef hash; TP, two-phase food. ${ }^{\star} \mathrm{P}<0.001$ for differences among food types. $₫ \mathrm{P}=$ 0.005 for difference between stroke and control groups. 
Citation: Matsuo K, Yokoyama M, Gonzalez-Fernandez M, Saitoh E, Kagaya H, et al. (2015) Effects of Food Consistencies and Mastication on Bolus Transport and Swallow Initiation in Individuals with Hemispheric Stroke. J Neurol Neurophysiol 6: 269. doi: $10.4172 / 2155-9562.1000269$

Page 7 of 9

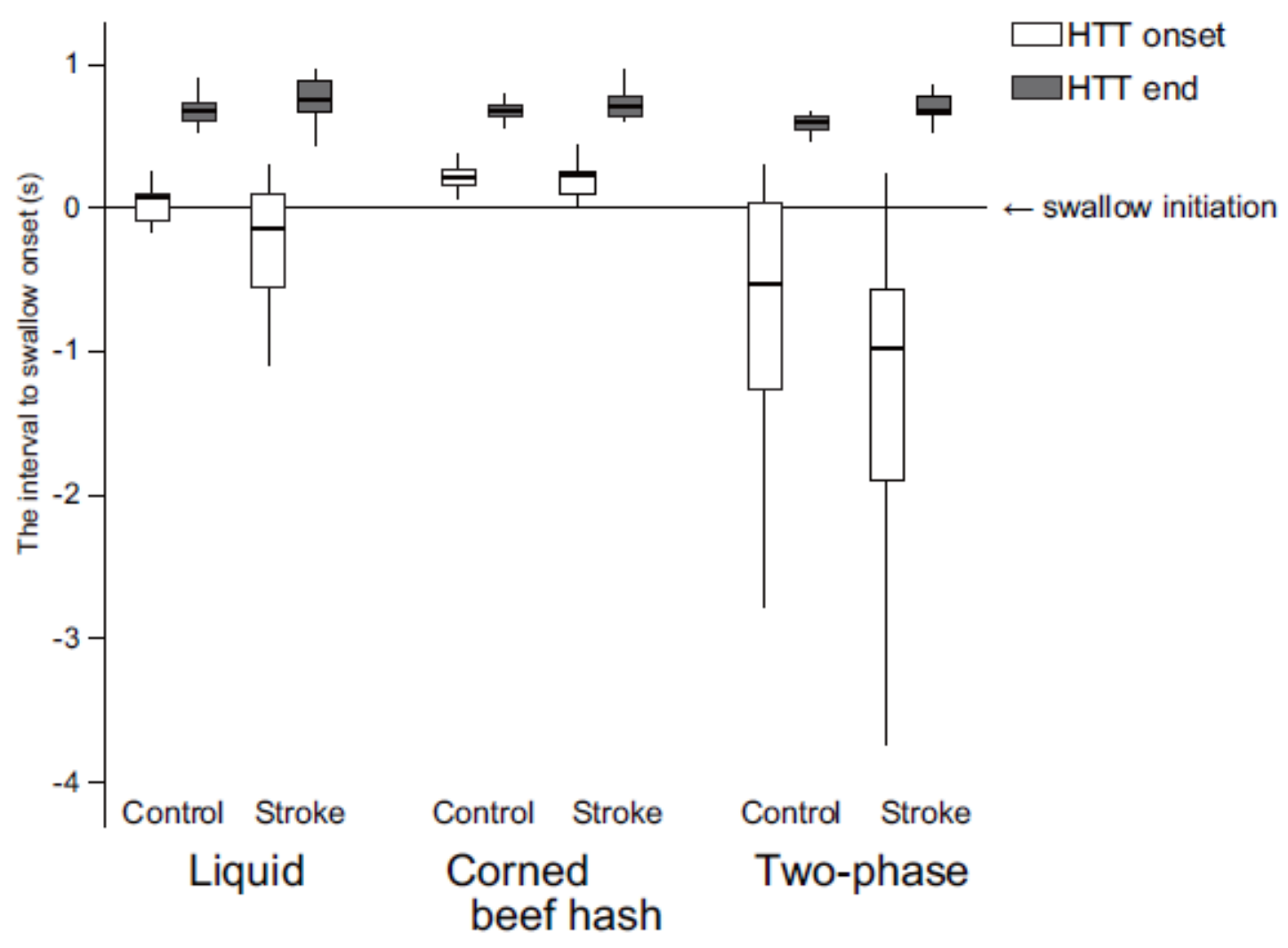

Figure 4: Intervals (mean $\pm \mathrm{SD}$ ) from swallow onset to the onset and end of HTT. Swallow onset was defined as time 0 . HTT durations were significantly longer in stroke survivors than controls, and significantly longer with the two-phase food than other foods $(\mathrm{P}<0.010)$. The interval from swallow onset to the end of the HTT did not differ significantly among food types $(\mathrm{P}=0.080)$. HTT, hypopharyngeal transit time

HTT duration was longer in the stroke group than in controls $(\mathrm{P}=$ 0.005). The time interval from the onset of the HTT until swallow onset and the interval from swallow onset to the end of HTT were also significantly longer in the stroke group than in controls $(\mathrm{P}<0.02$, Figure 4). HTT duration and the interval from the onset of the HTT until swallow onset was longer with two-phase samples than with other foods $(\mathrm{P}<0.01)$, but the interval from swallow onset until the end of the HTT did not differ significantly among food types $(\mathrm{P}=$ $0.080)$.

\section{Discussion}

The present study demonstrated that stroke alters the coordination of bolus transport and swallowing, particularly with foods containing a liquid phase. Our primary hypothesis was that the leading edge of the food entered the pharynx earlier, and was deeper in the pharynx at swallow onset in stroke survivors with dysphagia. Our results partially confirm the hypothesis; with liquid or two-phase samples, the leading edge of the food readily entered the pharynx before swallow initiation due to the low viscosity of the liquid phase while the bolus transport and pharyngeal accumulation during chewing did not differ with corned beef hash. These suggest that stroke may amplify the effect of thin liquid consistency on bolus transport and swallow initiation. In liquid bolus trials, the bolus was held in the mouth and then swallowed on command. When the swallow was initiated in the control group, the leading edge was at the bottom of the piriform sinus in $21 \%$ of subjects. This incidence was more than double (48\%) in the stroke group. With two-phase food, the sample was rarely held entirely in the mouth; since the tongue and palate move continuously during chewing, the fauces were open and the liquid component quickly entered the pharynx due to gravity. The leading edge of the food descended to the floor of the piriform sinus adjacent to the ariepiglottic folds in $44 \%$ of the control group and $76 \%$ of the stroke group. Thus, in stroke survivors, the ingesta were located in the piriform sinus for a longer time before swallow initiation. While eating, the larynx is open to the pharynx for breathing until swallow onset. Impairments in oral bolus control and pharyngeal swallow initiation allow the bolus to enter the hypopharynx prior to swallow initiation, thereby increasing the risk of aspiration.

The findings in this study also indicated a delay in the trigger for swallowing in stroke survivors for two-phase or liquid foods. Sensory stimuli from the oral cavity and pharynx are important factors in 
Citation: Matsuo K, Yokoyama M, Gonzalez-Fernandez M, Saitoh E, Kagaya H, et al. (2015) Effects of Food Consistencies and Mastication on Bolus Transport and Swallow Initiation in Individuals with Hemispheric Stroke. J Neurol Neurophysiol 6: 269. doi: $10.4172 / 2155-9562.1000269$

Page 8 of 9

triggering a swallow; prior studies suggest that individuals with dysphagia following stroke are more likely to have sensory deficits affecting these structures $[9,23]$. Although we did not directly examine pharyngeal sensation in our subjects, the sensory deficits that often accompany hemispheric stroke may have contributed to the altered coordination of bolus transport and pharyngeal swallow. It was reported that topical or transmucosal anesthesia of the oropharyngeal mucosa delayed swallow initiation of a liquid or chewed solid food bolus in healthy individuals, leading to aspiration in some [24-26]. Our findings suggest that in subjects with stroke, increased sensory stimuli covering a larger surface area in the foodway are necessary to trigger pharyngeal swallow.

Our secondary hypothesis was that deeper location of the leading edge of the food at swallow initiation was associated with an increased risk of aspiration. We found that PA scale scores were significantly higher when the leading edge reached the floor of the piriform sinus before swallow onset. This confirms our hypothesis that a deeper bolus position is associated with a risk of aspiration. Prior studies have reported a relationship between delayed swallow initiation of liquid and risk of aspiration in stroke survivors $[8,11,20,21]$. The present study extends this association to include two-phase food. Various mechanisms protect the airway during eating, drinking, and swallowing. These include respiratory inhibition (so-called "deglutitive apnea"), vocal fold closure, and physical separation of the airway from the foodway. Although this study did not analyze respiratory pattern, the laryngeal vestibule was open until swallow onset while the leading edge of the food entered the surrounding areas of the larynx. If sensory function is deficient in stroke survivors, thin liquids may enter deep into the hypopharynx and be associated with a significant risk of aspiration.

When eating two-phase food, the leading edge of the liquid phase commonly entered the hypopharynx before swallowing in both groups (74\% of the control and $90 \%$ of the stroke group). This is consistent with a previous study that described transport to the hypopharynx well before swallow onset in more than $60 \%$ of healthy young adults with two-phase food [17]. Despite liquid being present in the hypopharynx, aspiration was not observed in healthy adults in either study. Aspiration was seen only in stroke survivors and only when the leading edge reached the floor of the piriform sinus before swallow onset. Thus, the mechanisms that prevented aspiration in controls were clearly absent or ineffective in several of our stroke subjects. Stimulation of the hypopharynx with liquid administered gradually via a nasal tube can elicit a protective swallow, even in subjects instructed to suppress swallowing [27]. This swallow has been regarded as an airway protective response that is triggered when the bolus head reaches the bottom of the piriform sinus in normal individuals. In our healthy elderly controls, eating two-phase food, aspiration could have been prevented by a protective swallow. The mechanisms for preventing aspiration prior to swallow onset remain unclear and, in view of their importance in airway protection, warrant further study.

The progression of the leading edge in the oral cavity and pharynx during mastication and at swallow onset varied significantly by food type in both stroke and control groups. This may have reflected differences in bolus transport mechanisms that depend on food consistency. In a previous study of healthy young adult volunteers, the location of the leading edge at swallow onset was usually in the oral cavity for liquid, in the oropharynx for triturated solid food, and in the hypopharynx for two-phase food [17]. This transport of liquid phase to the hypopharynx is a gravity-dependent process [17]. When chewing, the tongue moves constantly and the soft palate elevates intermittently, opening of the fauces [28]. While eating two-phase food, the liquid phase leaks into the pharynx through the open fauces and quickly passes down to the hypopharynx. In contrast, the transport of triturated solid food to the oropharynx is an active tongue-driven process (stage II transport); the bolus usually accumulates in the oropharynx and rarely enters the hypopharynx before swallowing in both stroke and control subjects [29].

Consuming two-phase foods - either drinking liquid while chewing or eating food with a high liquid content - is commonplace in daily life. In the current study, the bolus head reached the bottom of the piriform sinus with exceptionally high frequency in dysphagic stroke survivors when eating two-phase food. The challenge of safely ingesting complex multi-textured foods is likely exacerbated in people with dysphagia after stroke. Since consuming two-phase food is associated with a high risk of aspiration, it may be advisable for individuals with dysphagia to avoid these consistencies. We further suggest that two-phase foods be included in instrumental examinations of swallowing.

\section{Conclusion}

The current study illustrates the significant impact of stroke on the progression of food down the foodway during mastication and at swallow initiation, and the major influence of food consistency. We found that the leading edge of the food moved deeper into the pharynx and remained there for a longer time before swallow onset in stroke survivors, especially when eating two-phase food or drinking liquid. A delay in pharyngeal swallow initiation increased the risk of aspiration. Pharyngeal stimulation by a food bolus either lower in the foodway or over a larger area may be necessary to elicit swallowing in individuals with dysphagia due to stroke.

\section{Acknowledgements}

This research was supported in part by USPHS Award R01 DC02123 from the National Institute on Deafness and other Communication Disorders and a Grant-in-Aid for Scientific Research (24792373) from the Ministry of Education, Culture, Sports, Science, and Technology, Japan.

\section{References}

1. Martino R, Foley N, Bhogal S, Diamant N, Speechley M, et al. (2005) Dysphagia after stroke: incidence, diagnosis, and pulmonary complications. Stroke 36: 2756-2763.

2. Hamdy S, Aziz Q, Rothwell JC, Crone R, Hughes D, et al. (1997) Explaining oropharyngeal dysphagia after unilateral hemispheric stroke. Lancet 350: 686-692.

3. Parker C, Power M, Hamdy S, Bowen A, Tyrrell P, et al. (2004) Awareness of dysphagia by patients following stroke predicts swallowing performance. Dysphagia 19: 28-35.

4. Finestone HM, Greene-Finestone LS, Wilson ES, Teasell RW (1995) Malnutrition in stroke patients on the rehabilitation service and at follow-up: prevalence and predictors. Arch Phys Med Rehabil 76: 310-316.

5. Hamdy S, Aziz Q, Rothwell JC, Power M, Singh KD, et al. (1998) Recovery of swallowing after dysphagic stroke relates to functional reorganization in the intact motor cortex. Gastroenterology 115: 1104-1112. 
Citation: Matsuo K, Yokoyama M, Gonzalez-Fernandez M, Saitoh E, Kagaya H, et al. (2015) Effects of Food Consistencies and Mastication on Bolus Transport and Swallow Initiation in Individuals with Hemispheric Stroke. J Neurol Neurophysiol 6: 269. doi: $10.4172 / 2155-9562.1000269$

Page 9 of 9

6. Hamdy S, Rothwell JC (1998) Gut feelings about recovery after stroke: the organization and reorganization of human swallowing motor cortex. Trends Neurosci 21: 278-282.

7. Daniels SK, Foundas AL (1999) Lesion localization in acute stroke patients with risk of aspiration. J Neuroimaging 9: 91-98.

8. Robbins J, Levine RL, Maser A, Rosenbek JC, Kempster GB (1993) Swallowing after unilateral stroke of the cerebral cortex. Arch Phys Med Rehabil 74: 1295-1300.

9. Aviv JE, Martin JH, Sacco RL, Zagar D, Diamond B, et al. (1996) Supraglottic and pharyngeal sensory abnormalities in stroke patients with dysphagia. Ann Otol Rhinol Laryngol 105: 92-97.

10. Hori K, Ono T, Iwata H, Nokubi T, Kumakura I (2005) Tongue pressure against hard palate during swallowing in post-stroke patients. Gerodontology 22: 227-233.

11. Power ML, Hamdy S, Singh S, Tyrrell PJ, Turnbull I, et al. (2007) Deglutitive laryngeal closure in stroke patients. J Neurol Neurosurg Psychiatry 78: 141-146.

12. Daniels SK1, Brailey K, Foundas AL (1999) Lingual discoordination and dysphagia following acute stroke: analyses of lesion localization. Dysphagia 14: 85-92.

13. Daniels SK, Foundas AL (2001) Swallowing physiology of sequential straw drinking. Dysphagia 16: 176-182.

14. Martin-Harris B, Brodsky MB, Michel Y, Lee FS, Walters B (2007) Delayed initiation of the pharyngeal swallow: normal variability in adult swallows. J Speech Lang Hear Res 50: 585-594.

15. Stephen JR, Taves DH, Smith RC, Martin RE (2005) Bolus location at the initiation of the pharyngeal stage of swallowing in healthy older adults. Dysphagia 20: 266-272.

16. Palmer JB, Rudin NJ, Lara G, Crompton AW (1992) Coordination of mastication and swallowing. Dysphagia 7: 187-200.

17. Saitoh E, Shibata S, Matsuo K, Baba M, Fujii W, et al. (2007) Chewing and food consistency: effects on bolus transport and swallow initiation. Dysphagia 22: 100-107.
18. Dua KS, Ren J, Bardan E, Xie P, Shaker R (1997) Coordination of deglutitive glottal function and pharyngeal bolus transit during normal eating. Gastroenterology 112: 73-83.

19. Hiiemae KM, Palmer JB (1999) Food transport and bolus formation during complete feeding sequences on foods of different initial consistency. Dysphagia 14: 31-42.

20. Kahrilas PJ, Lin S, Rademaker AW, Logemann JA (1997) Impaired deglutitive airway protection: a videofluoroscopic analysis of severity and mechanism. Gastroenterology 113: 1457-1464.

21. Clavé P, de Kraa M, Arreola V, Girvent M, Farré R, et al. (2006) The effect of bolus viscosity on swallowing function in neurogenic dysphagia. Aliment Pharmacol Ther 24: 1385-1394.

22. Rosenbek JC, Robbins JA, Roecker EB, Coyle JL, Wood JL (1996) A penetration-aspiration scale. Dysphagia 11: 93-98.

23. Kidd D, Lawson J, Nesbitt R, MacMahon J (1993) Aspiration in acute stroke: a clinical study with videofluoroscopy. Q J Med 86: 825-829.

24. Månsson I, Sandberg N (1975) Oro-pharyngeal sensitivity and elicitation of swallowing in man. Acta Otolaryngol 79: 140-145.

25. Ertekin C, Kiylioglu N, Tarlaci S, Keskin A, Aydogdu I (2000) Effect of mucosal anaesthesia on oropharyngeal swallowing. Neurogastroenterol Motil 12: 567-572.

26. Tei K, Yamazaki Y, Kobayashi M, Izumiyama Y, Ono M, et al. (2004) Effects of bilateral lingual and inferior alveolar nerve anesthesia effects on masticatory function and early swallowing. Oral Surg Oral Med Oral Pathol Oral Radiol Endod 97: 553-558.

27. Pouderoux P, Logemann JA, Kahrilas PJ (1996) Pharyngeal swallowing elicited by fluid infusion: role of volition and vallecular containment. Am J Physiol 270: G347-354.

28. Matsuo K, Hiiemae KM, Palmer JB (2005) Cyclic motion of the soft palate in feeding. J Dent Res 84: 39-42.

29. Palmer JB (1998) Bolus aggregation in the oropharynx does not depend on gravity. Arch Phys Med Rehabil 79: 691-696. 